\title{
BMI open The impact of smoke-free legislation on fetal, infant and child health: a systematic review and meta-analysis protocol
}

\author{
Jasper V Been, ${ }^{1,2}$ Ulugbek Nurmatov, ${ }^{2}$ Constant $P$ van Schayck, ${ }^{2,3}$ Aziz Sheikh ${ }^{2,3}$
}

To cite: Been JV,

Nurmatov U, van Schayck CP, et al. The impact of smokefree legislation on fetal, infant and child health: a systematic review and meta-analysis protocol. BMJ Open 2013;3: e002261. doi:10.1136/ bmjopen-2012-002261

- Prepublication history and additional material for this paper are available online. To view these files please visit the journal online (http://dx.doi.org/10.1136/ bmjopen-2012-002261).

Received 24 October 2012 Revised 24 December 2012 Accepted 17 January 2013

This final article is available for use under the terms of the Creative Commons Attribution Non-Commercial 2.0 Licence; see http://bmjopen.bmj.com

${ }^{1}$ Department of Paediatrics, School for Public Health and Primary Care (CAPHRI), Maastricht University Medical Centre, Maastricht,

The Netherlands

${ }^{2}$ Allergy \& Respiratory Research Group, Centre for Population Health Sciences, The University of Edinburgh Medical School, Edinburgh, UK

${ }^{3}$ Department of General Practice, School for Public Health and Primary Care (CAPHRI), Maastricht University Medical Centre, Maastricht, The Netherlands

Correspondence to Jasper V Been; jasper.been@mumc.nl

\section{ABSTRACT}

Introduction: Second-hand smoke (SHS) exposure is estimated to kill 600000 people worldwide annually. The WHO recommends that smoke-free indoor public environments are enforced through national legislation. Such regulations have been shown to reduce SHS exposure and, consequently, respiratory and cardiovascular morbidity. Evidence of particular health benefit in children is now emerging, including reductions in low birthweight deliveries, preterm birth and asthma exacerbations. We aim to comprehensively assess the impact of smoke-free legislation on fetal, infant and childhood outcomes. This can inform further development and implementation of global policy and strategies to reduce early life SHS exposure.

Methods: Two authors will search online databases (1975-present; no language restrictions) of published and unpublished/in-progress studies, and references and citations to articles of interest. We will consult experts in the field to identify additional studies. Studies should describe associations between comprehensive or partial smoking bans in public places and health outcomes among children (0-12 years): stillbirth, preterm birth, low birth weight, small for gestational age, perinatal mortality, congenital anomalies, bronchopulmonary dysplasia, upper and lower respiratory infections and wheezing disorders including asthma. The Cochrane Effectiveness Practice and Organisational Care (EPOC)-defined study designs are eligible. Study quality will be assessed using the Cochrane 7domain-based evaluation for randomised and clinical trials, and EPOC criteria for quasiexperimental studies. Data will be extracted by two reviewers and presented in tabular and narrative form. Metaanalysis will be undertaken using random-effects models, and generic inverse variance analysis for adjusted effect estimates. We will report sensitivity analyses according to study quality and design characteristics, and subgroup analyses according to coverage of ban, age group and parental/maternal smoking status. Publication bias will be assessed.

Ethics and dissemination: Ethics assessment is not required.

Results: Will be presented in one manuscript. The protocol is registered with PROSPERO, registration number CRD42013003522.

\section{ARTICLE SUMMARY}

Article focus

- Second-hand smoke exposure is associated with a range of adverse health outcomes among infants and children.

- Smoke-free legislation effectively reduces exposure to second-hand smoke and evidence for particular benefits for child health are now emerging.

- A comprehensive estimate of the effects of smoke-free legislation on perinatal and paediatric health is currently lacking.

Key messages

- We will undertake a systematic review and metaanalysis to comprehensively assess the impact of smoke-free legislation on perinatal and paediatric health.

- The results can inform the development and implementation of global policy and strategies to reduce second-hand smoke exposure in early life.

Strengths and limitations of this study

- We will interrogate a large number of online databases using an inclusive search strategy to identify the relevant literature. All Cochrane Effective Practice and Organisation of Care (EPOC) designs will be eligible for inclusion. Published guidelines will direct quality assessment and reporting.

\section{INTRODUCTION}

Tobacco use kills more than five million people annually, making it the leading global cause of preventable death. ${ }^{1}$ It is estimated that secondhand smoke (SHS) exposure kills an additional 600000 people worldwide each year, including 165000 children under 15 years. ${ }^{1}{ }^{2}$ Among non-smoking adults, SHS exposure furthermore increases the incidence of asthma, lung cancer and ischaemic heart disease. ${ }^{2}$ In an attempt to reduce this substantial burden on second-hand or passive smokers, the WHO has recommended that smoke-free indoor public environments are enforced through national legislation and that educational strategies are pursued in parallel to reduce SHS exposure in the home. ${ }^{3}$ Studies have since shown that 
smoking bans effectively reduce SHS exposure, even in the absence of an overall decline in smoking prevalence in the population. ${ }^{4}$ More importantly, consistent health effects have been reported in a recent Cochrane review summarising 25 studies including reductions in respiratory symptoms, sensory symptoms and admissions for acute myocardial infarction (AMI). ${ }^{4}$ These effects have since been reproduced by others, ${ }^{5-7}$ while additional studies also demonstrated reductions in sudden cardiac arrest and mortality from AMI in response to implementation of smokefree legislation. ${ }^{8} 9$

As developing individuals, children are particularly vulnerable to the negative effects of SHS, which may even ensue before birth. ${ }^{10-12}$ Furthermore, they are unable to influence their own degree of exposure. Antenatal SHS exposure puts unborn babies at risk for stillbirth, ${ }^{13}$ preterm delivery, ${ }^{14}$ growth retardation, ${ }^{12}{ }^{15}$ congenital anomalies, ${ }^{15} 16$ bronchopulmonary dysplasia ${ }^{17}$ and respiratory infections and asthma in childhood. ${ }^{11}{ }^{18}$ Worldwide, at least $40 \%$ of children are regularly exposed to SHS after birth, additionally predisposing them to upper and lower respiratory infections as well as asthma. ${ }^{2}$ Children thus bear an important part of the disease burden associated with SHS and are likely to particularly benefit from restrictive legislation. Indeed, several recent studies provide evidence for beneficial effects of smoke-free laws on infant and child health. Epidemiological evaluations of the 2006 Scottish smoking ban have demonstrated reductions in low birth weight, preterm birth and childhood asthma hospitalisations following its introduction. ${ }^{19} 20$ These results have now been confirmed in several follow-up studies. ${ }^{21} 22$

Despite this increasing evidence for particular health benefits of smoke-free legislation in children, the currently available systematic reviews assessing its health effects in general have not included any studies on perinatal or paediatric outcomes. ${ }^{42324}$ A comprehensive estimate of the benefits associated with smoke-free legislation in newborns and children will inform the development and implementation of global policy and strategies to further reduce SHS exposure in this particularly vulnerable population. Therefore, we will undertake a systematic review and meta-analysis of studies on fetal, infant and child-health outcomes related to the introduction of smoke-free legislation in order to obtain the most comprehensive assessment to date of its effectiveness in improving the health of babies and children worldwide.

\section{METHODS AND ANALYSIS}

Design

Systematic review and meta-analysis.

\section{Eligibility criteria}

Types of interventions

- Comprehensive (eg, bars, restaurants and working space) or partial (eg, working space only) smoking ban in public places at the national, state, city or community level.

Types of studies

- In keeping with the Cochrane Effective Practice and Organisation of Care (EPOC) guidelines that have set the standard for reviews of interventions designed to improve delivery of effective health services, only the following study designs will be considered for inclusion: (cluster) randomised controlled trials (RCTs), controlled clinical trials (CCTs), quasiexperimental studies, controlled before-and-after studies, interrupted time series (ITS) analysis. ${ }^{25}$ For nonrandomised studies, comparisons may include either a similarly aged population evaluated in the time frame preceding the introduction of the smoking ban in the same region, or a similar population evaluated during the same time frame in an adjacent geographical area where a smoking ban was not in place.

- Modelling, case-control, cohort, cross-sectional and uncontrolled before-and-after studies are excluded, given the difficulty in attributing causation from such studies.

Types of participants

- Fetuses $>20$ weeks gestation

- Newborns $>20$ weeks gestation

- Children aged 0-12 years. In order to minimise the confounding effect of self-smoking, we will restrict our analyses to children aged 12 years and under.

Types of outcome measures

Outcome measures should preferably be reported or documented by a health worker; alternatively, parentreported outcomes, parent-reported physician diagnoses or diagnoses based on medication use or prescriptions (eg, inhaled corticosteroids as a surrogate for asthma diagnosis) are acceptable. Outcomes may be defined as absolute (eg, incidence) or relative disease occurrence (eg, relative risk and $\mathrm{OR}$ ), or by associated health facility use (eg, doctor or emergency department visits and hospitalisation). Outcomes of interest are selected based on their relevance to fetal, infant and/or paediatric health and their recognised association between antenatal and/or postnatal SHS exposure. In addition, selection of primary outcomes is based on the magnitude of their burden for paediatric health, as well as their recognised reduction after the introduction of smoke-free legislation as shown by at least one high-quality study.

- Primary outcomes

- Preterm birth (live-birth between the 20th and 37th weeks of gestation)

- Low birth weight $(<2500 \mathrm{~g})$

- Asthma (recurrent or persistent wheezing in children aged 5 years or older)

- Secondary outcomes

- Perinatal outcomes - Stillbirth (intrauterine death of a fetus $>20$ weeks gestational age)

- Early neonatal death $(<1$ week postnatally)

- Perinatal death (stillbirth + neonatal death) 
- Late neonatal death (death 7-28 days postnatally)

- Neonatal death (death 0-28 days postnatally)

- Very preterm birth (<32 weeks' gestational age)

- Very low birth weight $(<1500 \mathrm{~g})$

- Extremely low birth weight $(<1000 \mathrm{~g})$

- Small for gestational age (birth weight $<10$ th percentile for gravidity, ethnicity and sex)

- Congenital anomalies

- Bronchopulmonary dysplasia

- Childhood outcomes

- Upper respiratory, infectious (pooled)

- Coryza, pharyngitis, tonsillitis, laryngitis/tracheitis, sinusitis, acute otitis media, influenza

- Upper respiratory, non-infectious

- Otitis media with effusion

- Lower respiratory, infectious (pooled)

- Bronchitis/bronchiolitis, whooping cough, pneumonia

- Lower respiratory, non-infectious

Wheezing ( $\geq 2$ wheezing episodes in children aged 4 years or younger)

Chronic cough (cough lasting $>4$ weeks)

- Outcomes not included in the review

- Surrogates and intermediates for adverse outcome (eg, intima media thickness, blood pressure, antioxidant activity)

- Smoke-related behaviours (eg, teenage smoking, attitude towards smoking, stopping behaviour)

- Measures of smoke exposure (eg, smoke exposure in the home, environmental nicotine measures, cotinine levels)

- Economic data (costs, cost-effectiveness)

When outcome definitions used in selected reports differ from the criteria outlined above, two authors (JVB and $\mathrm{UN}$ ) will make a decision regarding their inclusion in any meta-analyses. This will be based on the degree of deviation from the defined outcome criteria, and the expected effect that this may have on the analyses. A third author will be consulted to resolve any disagreement. Additional sensitivity analyses will be considered to explore the effect of inclusion of different outcome definitions.

\section{Search methods}

- Eligible study reports will be identified as follows:

- Published work will be searched for in the following databases: Cochrane Library (CENTRAL), Medline, EMBASE, AMED, CAB, Global Health, CINAHL, WHO Global Health Library (in addition to MEDLINE covering AIM (AFRO), LILACS (AMRO/PAHO), IMEMR (EMRO), IMSEAR (SEARO), WPRIM (WPRO), WHOLIS (KMS), SciELO), IndMED, TRIP, ISI Web of Science, KoreaMed, Google Scholar

- In addition, reference lists of articles of interest and citations to included articles will be screened for additional eligible published studies
- Unpublished and in-progress studies will be identified from the following trial registries: ClinicalTrials.gov; ISRCTN Register; WHO International Clinical Trials Registry Platform; EU Clinical Trials Register; Current Controlled Trials; Australian New Zealand Clinical Trial Registry; Pan African Clinical Trials Registry; Chinese Clinical Trial Register; Clinical Trials Register India; Brazilian Clinical Trials Registry; Clinical Research Information Service, Republic of Korea; Cuban Public Registry of Clinical Trials; German Clinical Trials Register; Iranian Registry of Clinical Trials; The Netherlands' Trialregister; Sri Lanka Clinical Trials Registry; UMIN Clinical Trials Registry

- Expert consultation

- Search strategy: see online supplementary appendices 1 and 2

- Restrictions

- Time span: 1975-current (rationale: the first regional smoking ban was introduced in 1975 in the US state of Minnesota) ${ }^{26}$

- Language: none (for foreign language papers translations will be sought)

\section{Study selection}

Two authors (JVB and UN) will search databases and screen titles and abstracts for potentially eligible studies. Disagreement will be resolved by consensus, or arbitration involving a third author where necessary. Full text articles will be retrieved for selected studies, and two authors (JVB and UN) will assess whether these meet inclusion criteria. Disagreement will be resolved by discussion among reviewers, with referral to a third author if necessary. Reasons for exclusion of studies will be noted.

\section{Quality assessment and analysis}

Study quality will be assessed using the Cochrane handbook 7-domain-based evaluation for RCTs, quasi-RCTs and CCTs (Cochrane handbook, table 8.5.a). ${ }^{27}$ For controlled before-and-after studies and ITS analyses, EPOC guidelines will be used. ${ }^{28}$ We will grade each parameter of trial quality: $\mathrm{A}$, low risk of bias; $\mathrm{B}$, moderate risk of bias; C, high risk of bias and an overall assessment for each controlled trial using the same three criteria will be made. Risk of bias will be assessed in part by recording design features (assessed by a formal list in the Cochrane handbook, table 13.2.a) as well as by whether or not confounding is accounted for. ${ }^{27}$ The primary confounder considered is maternal or parental smoking. Documentation of maternal/parental smoking according to smoke-free legislation status will be assessed, as well as adjustment of the final analyses for a potential confounding effect of this variable. All assessments of study quality will be performed by two authors (JVB and $\mathrm{UN}$ ), with any disagreement resolved by consensus, or arbitration involving a third author where necessary. 


\section{Data extraction}

Data will be extracted from selected papers by two reviewers (JVB and $\mathrm{UN})$, with any disagreement resolved by consensus, or arbitration involving a third author where necessary. Corresponding authors of eligible studies will be contacted to clarify any ambiguities. The following information will be extracted:

1. Geographical setting (eg, country and city)

2. Reported study type (eg, RCT and quasiexperimental study)

3. Design features (assessed by a formal list in the Cochrane handbook, table 13.2.a). ${ }^{27}$

4. Eligible population size

5. Included population size/number of clusters + cluster sizes

6. Relevant demographic characteristics (including age)

7. Description of intervention (including locations where ban was in effect (eg, bars, workplace and government buildings) and level of enforcement)

8. Inclusion/exclusion criteria

9. Outcomes

10. Effect sizes (univariate + multivariate)

11. Confounders adjusted for (eg, parental smoking)

12. Bias assessment

13. Adverse effects

14. Follow-up rate and handling of dropouts

15. Follow-up period

\section{Data analysis}

Data will be presented in tabular and narrative forms. If possible, meta-analysis will be performed on similar studies reporting main, primary and secondary outcomes, and be presented in forest plots. Choice of the statistical tests used will depend on the nature of the outcome variable. We will apply a random effects model in all analyses, given the expected degree of heterogeneity in the population and design between studies. Heterogeneity will be assessed both qualitatively and quantitatively using the $\mathrm{I}^{2}$ statistic. Meta-analysis will not be undertaken when $\mathrm{I}^{2}$ is equal to or greater than $75 \%$. Where possible, adjusted effect estimates will be pooled in meta-analyses using generic inverse-variance analysis. Adjusted effect estimates derived from the most adjusted model in the original paper will be selected for these analyses. Point estimates and $95 \%$ CIs will be reported for all analyses.

Sensitivity analyses will be performed in subgroups of study quality and of design characteristics (randomised vs non-randomised; prospective vs retrospective). If possible, analyses will be performed in subgroups made according to the following defining parameters: setting of smoking restriction (comprehensive vs locationspecific (eg, working space, bars and restaurants)), age of study individuals (under 5 vs 5 years and older), smoking status in the home or maternal smoking for perinatal outcomes. For meta-analyses of adjusted effect estimates, an additional sensitivity analysis will be performed according to whether or not maternal or parental smoking was part of the adjusted model in the original study.

For any meta-analysis that includes 10 or more studies, publication bias will be assessed visually through Funnel plots and tested by Egger's regression test and Begg's rank correlation test. ${ }^{29} 30$ All statistical analyses will be performed using Stata.

\section{ETHICS AND DISSEMINATION \\ Ethical issues}

As no primary data collection will be undertaken, no additional formal ethical assessment and informed consent are required.

\section{Publication plan}

The systematic review protocol is registered with the PROSPERO International Prospective Register of Systematic Reviews (http://www.crd.york.ac.uk/prospero). Findings will be summarised in a single manuscript.

\section{Timeline}

Start date: 1 January 2013

Finishing date: 31 March 2014

Reporting date: 31 March 2014

Acknowledgements We thank Marshall Dozier for valuable feedback on our search strategy, and the Netherlands Asthma Foundation, the Maastricht University Medical Centre, the Thrasher Research Fund and the International Pediatric Research Foundation for funding this work.

Contributors JVB conceived the study and prepared the initial protocol draft. JVB, UN, CPvS and AS were involved in study design, protocol and manuscript development. JVB and UN designed the search strategy. All authors read and approved the final version of the manuscript.

Funding This work was supported by the Thrasher Research Fund Early Career Award NR-0166; The Netherlands Asthma Foundation Long Term Fellowship 3.4.12.128FE (JVB); Maastricht University Medical Centre Kootstra Talent Fellowship (JVB) and the International Pediatric Research Foundation Young Investigator Exchange Programme (JVB).

Competing interests None.

Provenance and peer review Not commissioned; externally peer reviewed.

\section{REFERENCES}

1. WHO. WHO report on the global tobacco epidemic. Implementing smoke-free environments, Geneva, Switzerland: 2009.

2. Oberg M, Jaakkola MS, Woodward A, et al. Worldwide burden of disease from exposure to second-hand smoke: a retrospective analysis of data from 192 countries. Lancet 2011;377:139-46.

3. WHO. Protection from exposure to second-hand smoke. Geneva, Switzerland: Policy recommendations, 2007.

4. Callinan JE, Clarke A, Doherty K, et al. Legislative smoking bans for reducing secondhand smoke exposure, smoking prevalence and tobacco consumption. Cochrane Database Syst Rev 2010;(4): CD005992.

5. Reijula JP, Johnsson TS, Kaleva PS, et al. Exposure to tobacco smoke and prevalence of symptoms decreased among Finnish restaurant workers after the smoke-free law. Am J Ind Med 2012;55:37-43.

6. Madureira J, Mendes A, Almeida S, et al. Positive impact of the Portuguese smoking law on respiratory health of restaurant workers. $J$ Toxicol Environ Health A 2012;75:776-87.

7. Sebrie EM, Sandoya E, Hyland A, et al. Hospital admissions for acute myocardial infarction before and after implementation of a comprehensive smoke-free policy in Uruguay. Tob Control. 
Published Online First: 15 February 2012. doi:10.1136/ tobaccocontrol-2011-050134

8. de Korte-de Boer D, Kotz D, Viechtbauer W, et al. Effect of smoke-free legislation on the incidence of sudden circulatory arrest in the Netherlands. Heart 2012;98:995-9.

9. Villalbi JR, Sanchez E, Benet J, et al. The extension of smoke-free areas and acute myocardial infarction mortality: before and after study. BMJ Open 2011;1:e000067.

10. Murray RL, Britton J, Leonardi-Bee J. Second hand smoke exposure and the risk of invasive menigococcal disease in children: systematic review and meta-analysis. BMC Public Health 2012;12:1062.

11. Burke $\mathrm{H}$, Leonardi-Bee J, Hashim A, et al. Prenatal and passive smoke exposure and incidence of asthma and wheeze: systematic review and meta-analysis. Pediatrics 2012;129:735-44.

12. Leonardi-Bee J, Smyth A, Britton J, et al. Environmental tobacco smoke and fetal health: systematic review and meta-analysis. Arch Dis Child 2008;93:F351-61.

13. Flenady V, Koopmans L, Middleton $\mathrm{P}$, et al. Major risk factors for stillbirth in high-income countries: a systematic review and meta-analysis. Lancet 2011;377:1331-40.

14. van den Berg G, van Eijsden M, Vrijkotte TG, et al. Educational inequalities in perinatal outcomes: the mediating effect of smoking and environmental tobacco exposure. PLOS ONE 2012;7:e37002.

15. Salmasi G, Grady R, Jones J, et al. Environmental tobacco smoke exposure and perinatal outcomes: a systematic review and meta-analyses. Acta Obst Gynecol Scand 2010;89:423-41.

16. Hackshaw A, Rodeck $\mathrm{C}$, Boniface $\mathrm{S}$. Maternal smoking in pregnancy and birth defects: a systematic review based on 173687 malformed cases and 11.7 million controls. Hum Reprod Update 2011:17:589-604.

17. Antonucci R, Contu P, Porcella A, et al. Intrauterine smoke exposure: a new risk factor for bronchopulmonary dysplasia? J Perinat Med 2004;32:272-7.
18. Jones LL, Hashim A, McKeever T, et al. Parental and household smoking and the increased risk of bronchitis, bronchiolitis and other lower respiratory infections in infancy: systematic review and meta-analysis. Respir Res 2011;12:5.

19. Mackay D, Haw S, Ayres JG, et al. Smoke-free legislation and hospitalizations for childhood asthma. N Engl J Med 2010;363:1139-45.

20. Mackay DF, Nelson SM, Haw SJ, et al. Impact of Scotland's smoke-free legislation on pregnancy complications: retrospective cohort study. PLOS Med 2012;9:e1001175.

21. Kabir Z, Clarke V, Conroy R, et al. Low birthweight and preterm birth rates 1 year before and after the Irish workplace smoking ban. BJOG 2009;116:1782-7.

22. Page RL, Slejko JF, Libby AM. A citywide smoking ban reduced maternal smoking and risk for preterm births: a Colorado natural experiment. J Womens Health 2012;21:621-7.

23. Mackay DF, Irfan MO, Haw S, et al. Meta-analysis of the effect of comprehensive smoke-free legislation on acute coronary events. Heart 2010;96:1525-30.

24. Meyers DG, Neuberger JS, He J. Cardiovascular effects on smoking in public places: a systematic review and meta-analysis. J Am Coll Cardiol 2009;54:1249-55.

25. http://epoc.cochrane.org/sites/epoc.cochrane.org/files/uploads/EPOC \%20Study\%20Designs\%20About.pdf (accessed 8 Oct 2012).

26. http://www.health.state.mn.us/divs/eh/indoorair/mciaa/ftb/mciaa.pdf (accessed 8 Oct 2012).

27. http://www.cochrane-handbook.org/ (accessed 8 2012).

28. http://epoc.cochrane.org/sites/epoc.cochrane.org/files/uploads/ datacollectionchecklist.pdf (accessed 8 Oct 2012)

29. Egger M, Davey Smith G, Schneider M, et al. Bias in meta-analysis detected by a simple, graphical test. BMJ 1997;315:629-34.

30. Begg CB, Mazumdar M. Operating characteristics of a rank correlation test for publication bias. Biometrics 1994;50:1088-101. 